\title{
On semi-convergence of modified HSS iteration methods
}

\author{
Fang Chen • Qing-Quan Liu
}

Received: 15 August 2012 / Accepted: 23 November 2012 / Published online: 21 December 2012

(C) Springer Science+Business Media New York 2012

\begin{abstract}
We discuss semi-convergence of the modified Hermitian and skewHermitian splitting (MHSS) iteration method for solving a broad class of complex symmetric singular linear systems. The semi-convergence theory of the MHSS iteration method is established. In addition, numerical examples show the effectiveness of the MHSS iteration method when it is used as a solver or as a preconditioner (for the restarted GMRES method).
\end{abstract}

Keywords Complex symmetric matrix - Modified Hermitian and skew-Hermitian splitting $\cdot$ Iteration method $\cdot$ Semi-convergence

Mathematics Subject Classifications (2010) 65F10 - 65F50 - 65N22; CR: G1.3

\section{Introduction}

Consider an iterative solution of the system of linear equations

$$
A x=b, \quad A \in \mathbb{C}^{n \times n} \quad \text { and } \quad x, b \in \mathbb{C}^{n},
$$

where $A \in \mathbb{C}^{n \times n}$ is a complex symmetric matrix of the form

$$
A=W+\text { i } T
$$

F. Chen $(\bowtie) \cdot$ Q.-Q. Liu

Key Laboratory for Mechanics in Fluid Solid Coupling Systems, Institute of Mechanics, Chinese Academy of Sciences, Beijing, 100190, People's Republic of China e-mail: chenfreesky@gmail.com

Q.-Q. Liu

e-mail: qqliu@imech.ac.cn 
$W, T \in \mathbb{R}^{n \times n}$ are real symmetric matrices, and $\mathrm{i}=\sqrt{-1}$ is the imaginary unit. Moreover, we assume $T \neq 0$, which makes the matrix $A$ be a non-Hermitian matrix.

A complex symmetric matrix $A$ has the real and imaginary part splitting

$$
A=W+\mathrm{i} T
$$

and the Hermitian and skew-Hermitian splitting (HSS)

$$
A=H+S,
$$

where

$$
H=\frac{1}{2}\left(A+A^{*}\right)=W \quad \text { and } \quad S=\frac{1}{2}\left(A-A^{*}\right)=\mathrm{i} T .
$$

Bai, Golub and Ng established the Hermitian and skew-Hermitian splitting iteration method in [7]. Then this class of iteration methods has got in deep research on itself and its applications [3, 6, 8, 9, 12, 13, 16]. Complex symmetric linear system is a difficult problem but arises in a number of applications such as wave propagation, diffuse optical tomography, quantum mechanics, electromagnetism, molecular scattering, and structural dynamics. In recent years, there have been some other approaches applied to realistic problems $[2,4,5,10]$. The complex system of linear equations never leave our sight $[1,2,10,12,17]$. Based on the HSS iteration method, and the real and imaginary part splitting of the complex symmetric matrix $A \in$ $\mathbb{C}^{n \times n}$, Bai, Benzi and Chen established the modified Hermitian and skew-Hermitian splitting (MHSS) iteration method [4]. The MHSS iteration method is as follows.

The modified HSS iteration method Let $x^{(0)} \in \mathbb{C}^{n}$ be an arbitrary initial guess. For $k=0,1,2, \ldots$ until the sequence of iterates $\left\{x^{(k)}\right\}$ converges, compute the next iterate $x^{(k+1)}$ according to the following procedure:

$$
\left\{\begin{array}{l}
(\alpha I+W) x^{\left(k+\frac{1}{2}\right)}=(\alpha I-\mathrm{i} T) x^{(k)}+b, \\
(\alpha I+T) x^{(k+1)}=(\alpha I+\mathrm{i} W) x^{\left(k+\frac{1}{2}\right)}-\mathrm{i} b,
\end{array}\right.
$$

where $\alpha$ is a given positive constant and $I \in \mathbb{R}^{n \times n}$ represents the identity matrix.

When $W \in \mathbb{R}^{n \times n}$ and $T \in \mathbb{R}^{n \times n}$ are symmetric positive semidefinite, at least one of them is symmetric positive definite (or $\operatorname{null}(W) \cap \operatorname{null}(T)=\{0\})$, and $\alpha \in$ $\mathbb{R}$ is positive, the MHSS iteration method converges unconditionally to the exact solution of the system of linear equations (1). Furthermore, Bai, Benzi and Chen proposed a preconditioned MHSS (PMHSS) iteration method for a class of complex symmetric system of linear equations [5]. The numerical experiments in [5] showed that the PMHSS iteration method is meshsize-independent and parameter-insensitive for the tested numerical examples. The authors also obtained upper bounds for the contraction factors of the MHSS and the PMHSS iteration methods, but they could not give the optimal parameters. 
In this paper, we prove that the MHSS iteration method is semi-convergent for singular systems of linear equations (1). We also derive an upper bound for the semiconvergence factor of the MHSS iteration method, which depends on the largest and the smallest nonzero eigenvalues of the matrices $W$ and $T$. At last, we use two examples to show the effectiveness of the MHSS iteration method.

\section{The semi-convergence of the MHSS iteration method}

Firstly, we give the standard form of the MHSS iteration method. According to the MHSS iteration method (2), the coefficient matrix $A$ has the splitting

$$
A=B(\alpha)-C(\alpha)
$$

where

$$
B(\alpha)=\frac{1+\mathrm{i}}{2 \alpha}(\alpha I+W)(\alpha I+T)
$$

and

$$
C(\alpha)=\frac{1+\mathrm{i}}{2 \alpha}(\alpha I+\mathrm{i} W)(\alpha I-\mathrm{i} T) .
$$

Based on this splitting, we easily have the following standard form of the MHSS iteration method:

$$
x^{(k+1)}=M(\alpha) x^{(k)}+G(\alpha) b, \quad k=0,1,2, \ldots,
$$

where

$$
M(\alpha)=B(\alpha)^{-1} C(\alpha)=(\alpha I+T)^{-1}(\alpha I+\mathrm{i} W)(\alpha I+W)^{-1}(\alpha I-\mathrm{i} T)
$$

and

$$
G(\alpha)=B(\alpha)^{-1}=\alpha(1-\mathrm{i})(\alpha I+T)^{-1}(\alpha I+W)^{-1} .
$$

We call $M(\alpha)$ the iteration matrix of the MHSS iteration method and $B(\alpha)$ the MHSS preconditioner.

Next, we introduce some elementary concepts about the semi-convergence of an iteration method. Note that the convergence and semi-convergence of an iteration method can be found in $[11,14]$.

Lemma 2.1 $[3,11]$ Assume that $A$ is a singular matrix, and has the splitting $A=$ $B-C$, where $B$ is a nonsingular matrix. We can define an iteration method $x^{(k+1)}=$ $B^{-1} C x^{(k)}+B^{-1} f(k=0,1,2, \ldots)$ for the systems of linear equations $A x=f$. The necessary and sufficient conditions for guaranteeing the semi-convergence of this iteration method are as follows:

(i) the elementary divisors of the iteration matrix $M=B^{-1} C$ associated with its eigenvalue $\lambda=1$ are linear;

(ii) if $\lambda \in \sigma(M)$ and $|\lambda|=1$, then $\lambda=1$, i.e., $v(M)<1$, where

$$
\nu(M) \equiv \max \{|\lambda| \mid \lambda \in \sigma(M), \quad \lambda \neq 1\} .
$$


We know that when $A$ is a singular matrix, the matrix $M$ must have an eigenvalue 1 . In this case, the spectral radius of the iteration matrix is equal to 1 , i.e., $\rho(M)=1$. Therefore, when the iteration method is semi-convergent, $v(M)$ is called its semi-convergence factor.

According to the characteristic of the complex symmetric matrix $A$, from [3] we have the following lemma and theorem.

Lemma 2.2 [3] Let $A \in \mathbb{C}^{n \times n}$ be a singular matrix, and $W$ and $T$ be its real and imaginary parts, respectively. Let $W$ and $T$ be symmetric positive semidefinite. Then

$$
\operatorname{null}(A)=\operatorname{null}(W) \cap \operatorname{null}(T) .
$$

Theorem 2.1 Let $A \in \mathbb{C}^{n \times n}$ be a singular matrix, and $W$ and $T$ be its real and imaginary parts, respectively. Let $W$ and $T$ be symmetric positive semidefinite. Denote by $M(\alpha)$ the iteration matrix of the MHSS iteration method defined by (3). Then the index of the matrix $I-M(\alpha)$ is 1 .

Theorem 2.1 shows that the elementary divisors of the matrix $M(\alpha)$ associated with its eigenvalue 1 are linear. So the MHSS iteration method satisfies the condition (i) about the semi-convergence. Now we give the following theorem.

Theorem 2.2 Let $A \in \mathbb{C}^{n \times n}$ be a singular matrix, and $W$ and $T$ be its real and imaginary parts, respectively. Let $W$ and $T$ be symmetric positive semidefinite. Then, for any initial vector $x^{(0)} \in \mathbb{C}^{n}$, the MHSS iteration method is semi-convergent to a solution of the singular system of linear equations (1) or, say, $v(M(\alpha))<1$, for any positive constant $\alpha$.

Proof We only need to prove that the condition (ii) of the semi-convergence is satisfied.

Let

$$
\begin{aligned}
\tilde{M}(\alpha) & =(\alpha I-\mathrm{i} T) M(\alpha)(\alpha I-\mathrm{i} T)^{-1} \\
& =(\alpha I-\mathrm{i} T)(\alpha I+T)^{-1}(\alpha I+\mathrm{i} W)(\alpha I+W)^{-1} .
\end{aligned}
$$

Then $M(\alpha)$ is similar to $\tilde{M}(\alpha)$ and we only need to prove $v(\tilde{M}(\alpha))<1$.

Let $\tilde{M} x=\lambda x$. We proceed the proof by discussing all cases.

(a) $x \in \operatorname{null}(A)$, or equivalently, $W x=T x=0$.

In this case we easily get $\tilde{M}(\alpha) x=x$. So $\lambda=1$. On the other hand, when $\tilde{M}(\alpha) x=x$ we have

$$
(\alpha I+\mathrm{i} W)(\alpha I+W)^{-1} x=(\alpha I-\mathrm{i} T)^{-1}(\alpha I+T) x .
$$

Because $W$ is symmetric positive semidefinite, we assume that $\operatorname{rank}(W)=r$. Then there is an orthogonal matrix $P$ such that

$$
W=P\left(\begin{array}{cc}
\Lambda_{W} & 0 \\
0 & 0
\end{array}\right) P^{T} .
$$


It follows that

$$
\begin{aligned}
(\alpha I+\mathrm{i} W)(\alpha I+W)^{-1} x= & P\left(\begin{array}{cc}
\alpha I_{r}+\mathrm{i} \Lambda_{W} & 0 \\
0 & \alpha I_{n-r}
\end{array}\right) \\
& \times\left(\begin{array}{cc}
\alpha I_{r}+\Lambda_{W} & 0 \\
0 & \alpha I_{n-r}
\end{array}\right)^{-1} P^{T} x \\
= & P\left(\begin{array}{cc}
\left(\alpha I_{r}+\mathrm{i} \Lambda_{W}\right)\left(\alpha I_{r}+\Lambda_{W}\right)^{-1} & 0 \\
0 & I_{n-r}
\end{array}\right) P^{T} x,
\end{aligned}
$$

where $I_{r} \in \mathbb{C}^{r \times r}$ and $I_{n-r} \in \mathbb{C}^{(n-r) \times(n-r)}$ represent the identity matrices. Furthermore, we obtain

$$
\left\|(\alpha I+\mathrm{i} W)(\alpha I+W)^{-1} x\right\|_{2} \leq\left\|P^{T} x\right\|_{2}=\|x\|_{2} .
$$

Similar to the analysis of the above process, we can obtain

$$
\left\|(\alpha I-\mathrm{i} T)^{-1}(\alpha I+T) x\right\|_{2} \geq\|x\|_{2} .
$$

The above inequalities must be equalities because of (4). It then follows that $W x=T x=0$, or equivalently,

$$
A x=0 .
$$

So the necessary and sufficient conditions for $\tilde{M} x=x$ is $x \in \operatorname{null}(A)$.

(b) $x \notin \operatorname{null}(A)$, or equivalently, $x \notin \operatorname{null}(W) \cap \operatorname{null}(T)$. In this case, it must hold $\lambda \neq 1$. There are three situations stated as follows:

(b1) $x \in \operatorname{null}(W)$ but $x \notin \operatorname{null}(T)$. From $\tilde{M} x=\lambda x$, we get

$$
(\lambda+\mathrm{i}) T x=(1-\lambda) \alpha x .
$$

Because $\lambda \neq 1$ and $x \neq 0$, it holds that $\lambda \neq-\mathrm{i}$. Let $\lambda=\hat{a}+\hat{b}$ i. Then we know that

$$
\frac{1-\lambda}{\lambda+\mathrm{i}}=\frac{\hat{a}(1-\hat{a})-\hat{b}(1+\hat{b})-(1-\hat{a}+\hat{b}) \mathrm{i}}{\hat{a}^{2}+(\hat{b}+1)^{2}} .
$$

According to the property of $T$, we have

$$
(\hat{a}-\hat{b})-\left(\hat{a}^{2}+\hat{b}^{2}\right) \geq 0, \quad 1-\hat{a}+\hat{b}=0 .
$$

From the above relationships, we know that $|\lambda|=1$ if and only if $\lambda=1$ or $\lambda=-\mathrm{i}$, which is impossible. So in this case $|\lambda|<1$ holds true.

$\left(\mathrm{b}_{2}\right) \quad x \in \operatorname{null}(T)$ but $x \notin \operatorname{null}(W)$. We can easily get $|\lambda|<1$ according to the proof of $\left(\mathrm{b}_{1}\right)$.

$\left(\mathrm{b}_{3}\right) \quad x \notin \operatorname{null}(W)$ and $x \notin \operatorname{null}(T)$. Let $\tilde{M} x=\lambda x$. Then we have $x^{*} x \lambda=x^{*} \tilde{M} x=x^{*}(\alpha I-\mathrm{i} T)(\alpha I+T)^{-1}(\alpha I+\mathrm{i} W)(\alpha I+W)^{-1} x$.

By direct computations, we obtain

$$
\begin{aligned}
\|x\|_{2}^{2}|\lambda| & \leq\left\|x^{*}(\alpha I-\mathrm{i} T)(\alpha I+T)^{-1}\right\|_{2}\left\|(\alpha I+\mathrm{i} W)(\alpha I+W)^{-1} x\right\|_{2} \\
& <\|x\|_{2} \cdot\|x\|_{2} .
\end{aligned}
$$

So $|\lambda|<1$. 
Now, we have demonstrated $v(\tilde{M}(\alpha))<1$, or in other words, the MHSS iteration method is semi-convergent to a solution of the singular system of linear equations (1).

Similar to Theorem 3.4 and Corollary 3.7 in [3], we can easily get the following theorem and corollary.

Theorem 2.3 Let $A \in \mathbb{C}^{n \times n}$ be a singular matrix, and $W$ and $T$ be its real and imaginary parts, respectively. Let $W$ and $T$ be symmetric positive semidefinite and $\operatorname{rank}(A)=\operatorname{rank}(W)$. Denote by $M(\alpha)$ the iteration matrix of the MHSS iteration method defined by (3). Then $A$ is orthogonally similar to $\hat{A} \oplus 0$ such that the real part $\hat{W}$ and the imaginary part $\hat{T}$ of $\hat{A}$ are symmetric positive definite and symmetric positive semidefinite, respectively, which is equivalent to that $\hat{A}$ is the compression of $A$ on the range space of $W$.

Corollary 2.1 Let $A \in \mathbb{C}^{n \times n}$ be a singular matrix, $W$ and $T$ be its real and imaginary parts, respectively. Let $W$ and $T$ be symmetric positive semidefinite matrices. Then the semi-convergence factor $v(M(\alpha))$ of the MHSS iteration method is bounded by

$$
\varphi(M(\alpha))=\sup _{x \in \operatorname{null}(A)^{\perp}} \frac{\left|x^{*}(\alpha I-\mathrm{i} T)(\alpha I+T)^{-1}(\alpha I+\mathrm{i} W)(\alpha I+W)^{-1} x\right|}{x^{*} x} .
$$

According to the above results, we can obtain another upper bound of the semiconvergence factor.

Corollary 2.2 Let $A \in \mathbb{C}^{n \times n}$ be a singular matrix, and $W$ and $T$ be its real and imaginary parts, respectively. Let $W$ and $T$ be symmetric positive semidefinite matrices, and $\tau_{\min }$ and $\tau_{\max }$ be the minimum and the maximum nonzero eigenvalues among all eigenvalues of the matrices $W$ and $T$. Then

$$
v(M(\alpha)) \leq \varphi(M(\alpha)) \leq \phi(\alpha),
$$

where

$$
\phi(\alpha)=\max _{\tau_{\min } \leq \tau \leq \tau_{\max }} \frac{\sqrt{\alpha^{2}+\tau^{2}}}{\alpha+\tau} .
$$

Moreover,

$$
\alpha_{\star}=\arg \min _{\alpha}\left\{\max _{\tau_{\min } \leq \tau \leq \tau_{\max }} \frac{\sqrt{\alpha^{2}+\tau^{2}}}{\alpha+\tau}\right\}=\sqrt{\tau_{\min } \tau_{\max }}
$$

and

$$
\phi\left(\alpha_{\star}\right)=\frac{\sqrt{\frac{\tau_{\max }}{\tau_{\min }}+1}}{\sqrt{\frac{\tau_{\max }}{\tau_{\min }}}+1} .
$$


Proof We immediately have

$$
\begin{aligned}
\varphi(M(\alpha)) & =\sup _{x \in \operatorname{null}(A)^{\perp}} \frac{\left|x^{*}(\alpha I-\mathrm{i} T)(\alpha I+T)^{-1}(\alpha I+\mathrm{i} W)(\alpha I+W)^{-1} x\right|}{x^{*} x} \\
& \leq \sup _{x \in \operatorname{null}(A)^{\perp}} \frac{\left\|x^{*}(\alpha I-\mathrm{i} T)(\alpha I+T)^{-1}\right\|_{2}\left\|(\alpha I+\mathrm{i} W)(\alpha I+W)^{-1} x\right\|_{2}}{\|x\|_{2}^{2}} \\
& =\sup _{x \in \operatorname{null}(A)^{\perp}} \frac{\left\|(\alpha I+T)^{-1}(\alpha I+\mathrm{i} T) x\right\|_{2}}{\|x\|_{2}} \cdot \frac{\left\|(\alpha I+\mathrm{i} W)(\alpha I+W)^{-1} x\right\|_{2}}{\|x\|_{2}} \\
& \left.\leq \max _{\mu \in \sigma(T) \backslash\{0\}} \frac{|\alpha+\mathrm{i} \mu|}{|\alpha+\mu|}, \max _{\gamma \in \sigma(W) \backslash\{0\}} \frac{|\alpha+\mathrm{i} \gamma|}{|\alpha+\gamma|}\right\} \\
& =\max _{\mu \in \sigma(T) \backslash\{0\}}\left\{\max _{\frac{\sqrt{\alpha^{2}+\mu^{2}}}{\alpha+\mu}}, \max _{\gamma \in \sigma(W) \backslash\{0\}} \frac{\sqrt{\alpha^{2}+\gamma^{2}}}{\alpha+\gamma}\right\} \\
& \leq \max _{\tau_{\min } \leq \tau \leq \tau_{\max }} \frac{\sqrt{\alpha^{2}+\tau^{2}}}{\alpha+\tau} \\
& =\phi(\alpha) \\
& <1 .
\end{aligned}
$$

By direct computations, we know

$$
\alpha_{\star}=\sqrt{\tau_{\min } \tau_{\max }} .
$$

\section{Numerical experiments}

In this section we use two examples to show the feasibility and effectiveness of the MHSS iteration method. In our implementations, the initial vector $x^{(0)}$ is chosen to be the zero vector and the iteration is terminated once the current iterate $x^{(k)}$ satisfies

$$
\frac{\left\|b-A x^{(k)}\right\|_{2}}{\left\|b-A x^{(0)}\right\|_{2}} \leq 10^{-6} \text {. }
$$

In addition, all codes were run in MATLAB in double precision and the experiments were performed on a personal computer with $3.20 \mathrm{GHz}$ central processing unit (Intel(R) Core(TM) i5 CPU), $3.42 \mathrm{G}$ memory and windows operating system. For all examples, we implemented the MHSS and the HSS iteration methods as linear solvers, and also as preconditioners for the GMRES(5) method. We use the experimentally found optimal iteration parameters $\alpha_{\exp }$ of the MHSS and the HSS iteration methods, as well as the MHSS- and the HSS-preconditioned GMRES(5) iteration methods, which minimize the numbers of iteration steps; see Tables 1, 2 and 5. If the optimal iteration parameters form intervals, we further optimize it according to the lest computing times; see Tables 3, 4 and 6. 
Table 1 The experimentally found optimal iteration parameters $\alpha_{\exp }$ for MHSS and HSS iteration methods for Example 3.1

\begin{tabular}{|c|c|c|c|c|c|c|}
\hline \multirow[t]{2}{*}{$\gamma / m$} & \multicolumn{3}{|l|}{ MHSS } & \multicolumn{3}{|l|}{ HSS } \\
\hline & 64 & 80 & 96 & 64 & 80 & 96 \\
\hline $10^{1}$ & 0.09 & {$[0.06,0.07]$} & 0.05 & 0.23 & 0.19 & 0.16 \\
\hline $10^{2}$ & {$[0.33,0.35]$} & 0.24 & {$[0.18,0.19]$} & 0.22 & 0.18 & 0.15 \\
\hline $10^{3}$ & {$[1.24,1.39]$} & {$[0.90,0.98]$} & {$[0.70,0.73]$} & {$[0.21,0.22]$} & 0.18 & 0.15 \\
\hline $10^{4}$ & {$[0.51,1.12]$} & {$[0.62,0.67]$} & {$[0.66,0.85]$} & {$[0.21,0.22]$} & 0.18 & 0.15 \\
\hline
\end{tabular}

Example 3.1 [3] We consider the singular linear system $A x=b$, with the coefficient matrix $A=W+\mathrm{i} T \in \mathbb{C}^{n \times n}$ being given by

$$
W=I \otimes V_{c}+V_{c} \otimes I \in \mathbb{R}^{n \times n}, \quad T=\frac{\gamma}{2 m}\left(I \otimes U_{c}+U_{c} \otimes I\right) \in \mathbb{R}^{n \times n},
$$

with

$$
\begin{aligned}
& V_{c}=V-\left(e_{1} e_{m}^{T}+e_{m} e_{1}^{T}\right) \in \mathbb{R}^{m \times m} \\
& U_{c}=U-\left(e_{1} e_{m-1}^{T}+e_{m-1} e_{1}^{T}+e_{a} e_{m}^{T}+e_{m} e_{a}^{T}\right) \in \mathbb{R}^{m \times m}
\end{aligned}
$$

and

$$
\begin{aligned}
V & =\operatorname{tridiag}(-1,2,-1) \in \mathbb{R}^{m \times m}, \\
U & =\text { pentadiag }(-1,-1,4,-1,-1) \in \mathbb{R}^{m \times m}, \\
e_{1} & =(1,0, \ldots, 0) \in \mathbb{R}^{m}, \\
e_{m-1} & =(0, \ldots, 0,1,0) \in \mathbb{R}^{m}, \\
e_{m} & =(0, \ldots, 0,1) \in \mathbb{R}^{m}, \\
e_{a} & =(1,1,0, \ldots, 0) \in \mathbb{R}^{m} .
\end{aligned}
$$

\begin{tabular}{|c|c|c|c|c|c|c|}
\hline \multirow[t]{2}{*}{$\gamma / m$} & \multicolumn{3}{|c|}{ MHSS-GMRES(5) } & \multicolumn{3}{|c|}{ HSS-GMRES(5) } \\
\hline & 64 & 80 & 96 & 64 & 80 & 96 \\
\hline $10^{1}$ & 0.07 & {$[0.05,0.06]$} & 0.04 & 0.18 & {$[0.09,0.11]$} & 0.06 \\
\hline $10^{2}$ & {$[0.35,0.39]$} & 0.22 & {$[0.14,0.17]$} & {$[0.57,0.67]$} & {$[0.10,0.11]$} & 0.20 \\
\hline $10^{3}$ & {$[1.05,1.54]$} & {$[0.77,0.96]$} & {$[0.64,0.73]$} & {$[0.79,1.26]$} & {$[2.98,3.24]$} & {$[0.37,0.49]$} \\
\hline $10^{4}$ & {$[3.81,14.94]$} & {$[3.22,7.80]$} & {$[3.90,4.08]$} & {$[3.61,8.41]$} & {$[2.06,4.56]$} & {$[2.13,2.49]$} \\
\hline
\end{tabular}

The right-hand side vector $b$ is defined as $b=A x_{\star}$, with $x_{\star}=(1,2, \ldots, n)^{T} \in \mathbb{R}^{n}$.

This example is a constructed system of linear equations, but it has special features $[3,15]$. In Tables 1 and 2, we list the experimentally found optimal iteration parameters $\alpha_{\exp }$ of the MHSS and the HSS iteration methods, as well as the MHSS- and

Table 2 The experimentally found optimal iteration parameters $\alpha_{\exp }$ for MHSS- and HSS-preconditioned GMRES(5) methods for Example 3.1 
Table 3 Numerical results for MHSS and HSS iteration methods for Example 3.1

\begin{tabular}{|c|c|c|c|c|c|c|c|c|c|}
\hline & \multirow[t]{2}{*}{$\gamma$} & \multicolumn{4}{|c|}{ MHSS } & \multicolumn{4}{|l|}{ HSS } \\
\hline & & $\alpha_{\exp }$ & IT & $\mathrm{CPU}$ & RES & $\alpha_{\exp }$ & IT & $\mathrm{CPU}$ & RES \\
\hline \multirow[t]{4}{*}{$m=64$} & $10^{1}$ & 0.09 & 73 & 0.877 & $8.96 \mathrm{e}-007$ & 0.23 & 117 & 3.635 & $9.60 \mathrm{e}-007$ \\
\hline & $10^{2}$ & 0.33 & 83 & 0.976 & $9.72 \mathrm{e}-007$ & 0.22 & 119 & 3.625 & $9.49 \mathrm{e}-007$ \\
\hline & $10^{3}$ & 1.33 & 49 & 0.599 & $8.18 \mathrm{e}-007$ & 0.21 & 119 & 3.522 & $9.69 \mathrm{e}-007$ \\
\hline & $10^{4}$ & 1.08 & 111 & 1.282 & $9.67 \mathrm{e}-007$ & 0.22 & 119 & 3.603 & $9.29 \mathrm{e}-007$ \\
\hline \multirow[t]{4}{*}{$m=80$} & $10^{1}$ & 0.07 & 86 & 1.855 & $9.06 \mathrm{e}-007$ & 0.19 & 144 & 8.445 & $9.45 \mathrm{e}-007$ \\
\hline & $10^{2}$ & 0.24 & 102 & 2.187 & $9.98 \mathrm{e}-007$ & 0.18 & 146 & 8.366 & $9.83 \mathrm{e}-007$ \\
\hline & $10^{3}$ & 0.98 & 60 & 1.303 & $9.65 \mathrm{e}-007$ & 0.18 & 146 & 8.383 & $9.84 \mathrm{e}-007$ \\
\hline & $10^{4}$ & 0.65 & 98 & 2.062 & $9.69 \mathrm{e}-007$ & 0.18 & 146 & 8.391 & $9.84 \mathrm{e}-007$ \\
\hline \multirow[t]{4}{*}{$m=96$} & $10^{1}$ & 0.05 & 91 & 2.864 & $9.17 \mathrm{e}-007$ & 0.16 & 170 & 16.806 & $9.47 \mathrm{e}-007$ \\
\hline & $10^{2}$ & 0.18 & 122 & 3.806 & $9.61 \mathrm{e}-007$ & 0.15 & 174 & 17.025 & $9.41 \mathrm{e}-007$ \\
\hline & $10^{3}$ & 0.70 & 72 & 2.481 & $9.94 \mathrm{e}-007$ & 0.15 & 172 & 16.656 & $9.80 \mathrm{e}-007$ \\
\hline & $10^{4}$ & 0.75 & 93 & 3.116 & $9.32 \mathrm{e}-007$ & 0.15 & 172 & 17.137 & $9.78 \mathrm{e}-007$ \\
\hline
\end{tabular}

the HSS-preconditioned GMRES(5) iteration methods for different $\gamma$ and $m$, respectively. The results show that all $\alpha_{\exp }$ with respect to MHSS are less than 1.39, the $\alpha_{\text {exp }}$ with respect to HSS are almost not changed for each fixed $m$, and the $\alpha_{\text {exp }}$ with respect to MHSS- and HSS-preconditioned GMRES(5) are easily form intervals.

In Tables 3 and 4 , we list the optimal parameters $\left(\alpha_{\exp }\right)$, the iteration steps (IT), the CPU times in seconds (CPU) and the relative residual errors (RES) for

Table 4 Numerical results for MHSS- and HSS-preconditioned GMRES(5) methods for Example 3.1

\begin{tabular}{|c|c|c|c|c|c|c|c|c|c|}
\hline & \multirow[t]{2}{*}{$\gamma$} & \multicolumn{4}{|c|}{ MHSS-GMRES(5) } & \multicolumn{4}{|c|}{ HSS-GMRES(5) } \\
\hline & & $\alpha_{\exp }$ & IT & $\mathrm{CPU}$ & RES & $\alpha_{\exp }$ & IT & $\mathrm{CPU}$ & RES \\
\hline \multirow[t]{4}{*}{$m=64$} & $10^{1}$ & 0.07 & 14 & 0.213 & $7.16 \mathrm{e}-007$ & 0.18 & 37 & 0.731 & $9.98 \mathrm{e}-007$ \\
\hline & $10^{2}$ & 0.35 & 19 & 0.275 & $9.91 \mathrm{e}-007$ & 0.67 & 50 & 0.962 & $9.90 \mathrm{e}-007$ \\
\hline & $10^{3}$ & 1.31 & 13 & 0.187 & $3.94 \mathrm{e}-007$ & 0.98 & 24 & 0.464 & $7.31 \mathrm{e}-007$ \\
\hline & $10^{4}$ & 6.19 & 8 & 0.113 & $2.31 \mathrm{e}-007$ & 4.68 & 11 & 0.222 & $6.88 \mathrm{e}-007$ \\
\hline \multirow[t]{4}{*}{$m=80$} & $10^{1}$ & 0.06 & 16 & 0.426 & $9.11 \mathrm{e}-007$ & 0.11 & 42 & 1.570 & $7.74 \mathrm{e}-007$ \\
\hline & $10^{2}$ & 0.22 & 22 & 0.579 & $9.58 \mathrm{e}-007$ & 0.10 & 69 & 2.572 & $9.97 e-007$ \\
\hline & $10^{3}$ & 0.87 & 15 & 0.373 & $6.37 \mathrm{e}-007$ & 2.98 & 34 & 1.242 & $9.78 \mathrm{e}-007$ \\
\hline & $10^{4}$ & 5.30 & 9 & 0.246 & $1.19 \mathrm{e}-007$ & 3.93 & 14 & 0.581 & $6.89 \mathrm{e}-007$ \\
\hline \multirow[t]{4}{*}{$m=96$} & $10^{1}$ & 0.04 & 17 & 0.656 & $4.96 \mathrm{e}-007$ & 0.06 & 49 & 3.092 & $9.25 \mathrm{e}-007$ \\
\hline & $10^{2}$ & 0.14 & 25 & 0.953 & $7.55 \mathrm{e}-007$ & 0.20 & 89 & 5.776 & $9.98 \mathrm{e}-007$ \\
\hline & $10^{3}$ & 0.69 & 17 & 0.657 & $7.45 \mathrm{e}-007$ & 0.40 & 43 & 2.762 & $8.70 \mathrm{e}-007$ \\
\hline & $10^{4}$ & 3.92 & 9 & 0.356 & $9.55 \mathrm{e}-007$ & 2.18 & 16 & 1.091 & $9.85 \mathrm{e}-007$ \\
\hline
\end{tabular}


the MHSS and the HSS iteration methods, as well as the MHSS- and the HSSpreconditioned GMRES(5) methods for different $\gamma$ and $m$. These results show that the iteration steps of the HSS iteration method are almost the same for each fixed $m$. So the HSS iteration method is not sensitive to $\gamma$. The iteration steps and the CPU times of the HSS iteration method are always larger than those of the MHSS iteration method. As preconditioners, the iteration steps and the CPU times of MHSSpreconditioned GMRES(5) method are less than those of the HSS-preconditioned GMRES(5) method. Hence, the MHSS iteration method outperforms the HSS iteration method in terms of iteration step and CPU time, and as preconditioners for GMRES(5) the MHSS performs much better than the HSS in terms of iteration step and CPU time, too.

Example 3.2 We consider the singular linear system $A x=b$, with the coefficient matrix $A=W+\mathrm{i} T \in \mathbb{C}^{n \times n}$ being given by

$$
W=\operatorname{tridiag}\left(c_{i-1}, a_{i}, c_{i}\right) \in \mathbb{R}^{n \times n}, \quad T=I \otimes V_{c}+V_{c} \otimes I \in \mathbb{R}^{n \times n},
$$

with

$$
V_{c}=V-\left(e_{1} e_{m}^{T}+e_{m} e_{1}^{T}\right) \in \mathbb{R}^{m \times m}
$$

and

$$
\begin{aligned}
V & =\operatorname{tridiag}(-1,2,-1) \in \mathbb{R}^{m \times m}, \\
e_{1} & =(1,0, \ldots, 0) \in \mathbb{R}^{m}, \\
e_{m} & =(0, \ldots, 0,1) \in \mathbb{R}^{m}, \\
a_{i} & =(1,3,5,7, \ldots, 2 n-3, n-1) \in \mathbb{R}^{n}, \\
c_{i} & =(-1,-2, \ldots,-(n-1)) \in \mathbb{R}^{n-1} .
\end{aligned}
$$

The right-hand side vector $b$ is defined as $b=A x_{\star}$, with $x_{\star}=(1,2, \ldots, n)^{T} \in \mathbb{R}^{n}$.

This example is also a constructed system of linear equations satisfying $(1, \ldots, 1)^{T} \in \operatorname{null}(W) \cap \operatorname{null}(T)$. In Table 5, we list the experimentally found optimal iteration parameters $\alpha_{\text {exp }}$ of the MHSS iteration method, as well as the MHSSand the HSS-preconditioned GMRES(5) iteration methods. We can not find the experimentally found optimal iteration parameters for the HSS iteration method when we set the interval $[0.01,1000]$ and the maximal iteration step 5000. For the MHSS iteration method, as well as the MHSS- and the HSS-preconditioned GMRES(5) iteration methods, the results in Table 5 show that all $\alpha_{\exp }$ are less than 0.40 and, moreover, $\alpha_{\text {exp }}$ becomes small with the growing of the $m$ for these three methods.

Table 5 The experimentally found optimal iteration parameters $\alpha_{\exp }$ for Example 3.2

\begin{tabular}{llll}
\hline Method & $m=64$ & $m=80$ & $m=96$ \\
\hline MHSS & 0.32 & 0.25 & 0.21 \\
HSS & - & - & - \\
MHSS-GMRES(5) & {$[0.39,0.40]$} & 0.30 & {$[0.24,0.26]$} \\
HSS-GMRES(5) & 0.26 & 0.20 & 0.14 \\
\hline
\end{tabular}


Table 6 Numerical results for Example 3.2

\begin{tabular}{|c|c|c|c|c|c|c|c|c|c|c|}
\hline \multirow[t]{2}{*}{$m$} & \multicolumn{3}{|c|}{ MHSS } & \multicolumn{4}{|c|}{ MHSS-GMRES(5) } & \multicolumn{3}{|c|}{ HSS-GMRES(5) } \\
\hline & IT & $\mathrm{CPU}$ & RES & $\alpha_{\exp }$ & IT & $\mathrm{CPU}$ & RES & IT & $\mathrm{CPU}$ & RES \\
\hline 64 & 385 & 1.461 & $9.82 \mathrm{e}-007$ & 0.40 & 218 & 0.863 & $8.29 \mathrm{e}-007$ & 1199 & 12.882 & $9.88 \mathrm{e}-007$ \\
\hline 80 & 489 & 3.223 & $9.90 \mathrm{e}-007$ & 0.30 & 266 & 2.075 & $9.02 \mathrm{e}-007$ & 1572 & 30.516 & $9.92 \mathrm{e}-007$ \\
\hline 96 & 595 & 5.691 & $9.79 \mathrm{e}-007$ & 0.24 & 309 & 3.594 & $9.95 \mathrm{e}-007$ & 1940 & 64.777 & $9.86 \mathrm{e}-007$ \\
\hline
\end{tabular}

In Table 6, we list the iteration steps, the CPU times and the relative residual errors for MHSS iteration method, as well as the MHSS- and the HSS-preconditioned GMRES(5) iteration methods. These results show that the iteration steps and the CPU times of each method become large with the growing of $m$. As preconditioners for GMRES(5) the MHSS-preconditioned GMRES(5) method has less iteration steps and CPU times than the HSS-preconditioned GMRES(5) method. Hence, the MHSS iteration method is superior to the HSS iteration method in both iteration step and CPU time, and as preconditioners for GMRES(5) MHSS outperforms HSS in iteration step and CPU time, too.

Acknowledgments This work is supported by the National Natural Science Funds of China for Distinguished Young Scholar (No. 10825211) and the Key project of Natural Science Foundation of China (No. 10932012).

\section{References}

1. Axelsson, O.: Iterative Solution Methods. Cambridge University Press, Cambridge (1996)

2. Axelsson, O., Kucherov, A.: Real valued iterative methods for solving complex symmetric linear systems. Numer. Linear Algebra Appl. 7, 197-218 (2000)

3. Bai, Z.-Z.: On semi-convergence of Hermitian and skew-Hermitian splitting methods for singular linear systems. Computing 89, 171-197 (2010)

4. Bai, Z.-Z., Benzi, M., Chen, F.: Modified HSS iteration methods for a class of complex symmetric linear systems. Computing 87, 93-111 (2010)

5. Bai, Z.-Z., Benzi, M., Chen, F.: On preconditioned MHSS iteration methods for complex symmetric linear systems. Numer. Algor. 56, 297-317 (2011)

6. Bai, Z.-Z., Golub, G.H., Li, C.-K.: Convergence properties of preconditioned Hermitian and skewHermitian splitting methods for non-Hermitian positive semidefinite matrices. Math. Comput. 76, 287-298 (2007)

7. Bai, Z.-Z., Golub, G.H., Ng, M.K.: Hermitian and skew-Hermitian splitting methods for nonHermitian positive definite linear systems. SIAM J. Matrix Anal. Appl. 24, 603-626 (2003)

8. Bai, Z.-Z., Golub, G.H., Ng, M.K.: On inexact Hermitian and skew-Hermitian splitting methods for non-Hermitian positive definite linear systems. Linear Algebra Appl. 428, 413-440 (2008)

9. Bai, Z.-Z., Golub, G.H., Pan, J.-Y.: Preconditioned Hermitian and skew-Hermitian splitting methods for non-Hermitian positive semidefinite linear systems. Numer. Math. 98, 1-32 (2004)

10. Benzi, M., Bertaccini, D.: Block preconditioning of real-valued iterative algorithms for complex linear systems. IMA J. Numer. Anal. 28, 598-618 (2008)

11. Berman, A., Plemmons, R.J.: Nonnegative Matrices in the Mathematical Sciences, Second Edition. SIAM, Philadelphia (1994)

12. Bertaccini, D.: Efficient preconditioning for sequences of parametric complex symmetric linear systems. Electron. Trans. Numer. Anal. 18, 49-64 (2004) 
13. Bertaccini, D., Golub, G.H., Capizzano, S.S., Possio, C.T.: Preconditioned HSS methods for the solution of non-Hermitian positive definite linear systems and applications to the discrete convectiondiffusion equation. Numer. Math. 99, 441-484 (2005)

14. Golub, G.H., van Loan, C.F.: Matrix Computations, Third Edition. The Johns Hopkins University Press, Baltimore and London (1996)

15. Gray, R.M.: Toeplitz and circulant matrices: a review. Foundations Trends Commun. Inform. Theory 2, 155-239 (2006)

16. Guo, X.-X., Wang, S.: Modified HSS iteration methods for a class of non-Hermitian positive-definite linear systems. Appl. Math. Comput. 218, 10122-10128 (2012)

17. Magolu, M., Made, M.: Incomplete factorization-based preconditionings for solving the Helmholtz equation. Int. J. Numer. Methods Eng. 50, 1077-1101 (2001) 